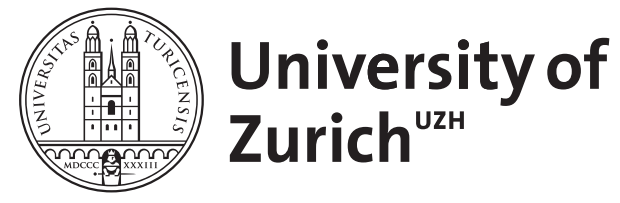

\title{
MAGI3-AKT3 fusion in breast cancer amended
}

Mosquera, Juan-Miguel ; Varma, Sonal ; Pauli, Chantal ; MacDonald, Theresa Y ; Yashinskie, Jossie J ; Varga, Zsuzsanna ; Sboner, Andrea ; Moch, Holger ; Rubin, Mark A ; Shin, Sandra J

DOI: https://doi.org/10.1038/nature14265

Posted at the Zurich Open Repository and Archive, University of Zurich ZORA URL: https://doi.org/10.5167/uzh-110350

Journal Article

Accepted Version

Originally published at:

Mosquera, Juan-Miguel; Varma, Sonal; Pauli, Chantal; MacDonald, Theresa Y; Yashinskie, Jossie J; Varga, Zsuzsanna; Sboner, Andrea; Moch, Holger; Rubin, Mark A; Shin, Sandra J (2015). MAGI3AKT3 fusion in breast cancer amended. Nature, 520(7547):E11-E12.

DOI: https://doi.org/10.1038/nature14265 


\section{MAG13-AKT3 fusion in breast cancer amended}

ARISING FROM S. Banerji et al. Nature 486, 405-409 (2012)

Banerji et al. ${ }^{1}$ described a novel $M A G I 3-A K T 3$ rearrangement in breast cancer, enriched in triple negative tumours. The report was highly encouraging as targeted therapies could potentially serve as a new and much needed option to treat this highly aggressive breast cancer subtype. We sought to confirm the presence of this rearrangement in 236 samples of triple negative breast cancer (TNBC) by using fluorescent in situ hybridization (FISH) and reverse transcriptionpolymerase chain reaction (RT-PCR), and in 84 additional cases from The Cancer Genome Atlas by using FusionSeq. No evidence of the fusion was found in any of the tumours studied. Our study confirms that $M A G I 3-A K T 3$ fusion is not a recurrent event in triple negative breast cancer, which should be acknowledged before considering the evaluation of targeted therapies in clinical trials. There is a Reply to this Brief Communication Arising by Pugh T. et al. Nature 520, http://dx.doi.org/10.1038/nature14266 (2015).

TNBC constitutes the majority of breast carcinomas of the basal-like molecular subtype, and is defined by absence of actionable therapeutic targets (ER, PR, HER-2). TNBC patients have a poor response to conventional breast cancer therapies ${ }^{2}$ and experience poor survival ${ }^{3}$. As such, molecular elucidation of these tumours is critical in the hopes of developing novel targeted therapies ${ }^{4}$.

Discovery of functionally recurrent gene rearrangements is a relatively new avenue in breast cancer (for example, MAST kinase and Notch gene families ${ }^{5}$ ). Banerji et al. reported a MAGI3-AKT3 gene fusion to be present in 7\% (5/72) of TNBC ${ }^{1}$. This balanced translocation results in a constitutive activation of AKT kinase, which can be countered using small molecule AKT inhibitors.

We aimed to determine the frequency of $M A G I 3-A K T 3$ fusion in 236 TNBCs represented in high-density tissue microarrays (see Table 1). Following previously described protocols ${ }^{6-8}$, FISH was performed using dual colour locus-specific probes for MAGI3 and AKT3. None of the cases showed either MAGI3 or AKT3 break-apart or fusion signals. To exclude the possibility of intra-tumour heterogeneity, multiple regions of full tumour sections were screened in a subset of 28 cases, all of which were also negative for break-apart and fusion signals (see Fig. 1).

Additionally, shorter primer sequences were designed to test a $187 \mathrm{bp}$ fusion product of intron 9 
of MAGI3 with intron 1 of $A K T 3$ in archival material. We performed RT-PCR of cDNA in 135 of these cases. No MAGI3-AKT3 fusion product was detected. Further, we investigated RNA-seq data from 84 TNBC cases from The Cancer Genome Atlas with FusionSeq. We did not find any evidence of MAGI3-AKT3 gene fusion in these cases either.

Our sample size has sufficient power to detect with $95 \%$ confidence gene rearrangements that would occur at a frequency of as low as $3 \%$. Based on our results, we can reliably conclude that MAGI3-AKT3 rearrangement is neither recurrent nor sub-clonal in TNBC. To assume that MAGI3-AKT3 fusion was a recurrent event in TNBC, Banerji et al. ${ }^{1}$ interrogated their tumours by using RT-PCR of cDNA followed by Sanger sequencing only. Confirmation at the genomic level by PCR of genomic DNA was performed exclusively in the index case. Hence, we favour that, with the exception of the index case, the sequenced RT-PCR products by Banerji et al. ${ }^{1}$ represent a post-transcriptional fusion event (trans-splicing), rather than a true genomic event.

Our patient cohort of mainly Caucasian women in contrast to those of Mexican and Vietnamese decent studied in Banerji et al. ${ }^{1}$ raises the possibility that this rearrangement may be population-enriched, a prospect that needs further study.

\section{Methods}

Locus specific probes were located at 1p (MAGI3: BAC 5' RP11-1133G15 and 3' RP11-1008I9) and 1q (AKT3: BAC 5' RP11-931B5 and 3' RP11-989N14). At least 150 nuclei per case were interrogated in tissue microarrays. In full sections, $\sim 2,000$ nuclei per slide were evaluated. FusionSeq ${ }^{9}$ is a robust computational tool to detect fusion transcripts in paired-end RNA-seq data $^{10-12}$. Reads were aligned to the human reference genome sequence (GRC37/hg19) using $\mathrm{STAR}^{13}$. PCR primers sequences are as follows. MAGI3 forward: $5^{\prime}-$

TGTCCTTGTTCGAGCATCAC-3', MAGI3 reverse: 5'-GAGGACACAGTTGCCATTGA-3', AKT3 forward: 5'-TGAAAGAAGGTTGGGTTCAGA-3', AKT3 reverse: 5'-

GCCACTGAAAAGTTGTTGAGG-3'. $P G K$ was used as control gene.

Figure 1 Absence of MAGI3-AKT3 fusion in triple negative breast cancer. a, Haematoxylin and eosin (H \& E) stained full section of a representative case of triple-negative breast cancer. b, c, Tissue microarrays (b) and multiple regions of the entire section (c) were interrogated by FISH (total of 236 cases). d, No break-apart signals for MAGI3 or AKT 3 were identified. No MAGI3AKT3 fusion product was detected by RT-PCR in a subset of 135 cases. 
Table 1: Clinico-pathologic characteristics of 236 triple negative breast cancers

\begin{tabular}{lll}
\hline Patient Age & $22-92$ years & \\
Tumour size & $0.3-7.4 \mathrm{~cm}$ & \\
& & $218^{*}$ \\
Number of tumour type casest & Invasive ductal carcinoma & 5 \\
& Invasive lobular carcinoma & 5 \\
& Metaplastic carcinoma & 8 \\
& Other & \\
& & $47.1 \%$ \\
Stage (percentage of cases) & Stage IA & $0.6 \%$ \\
& Stage IB & $0.6 \%$ \\
& Stage IC & $31.4 \%$ \\
& Stage IIA & $6.5 \%$ \\
& Stage IIB & $4.6 \%$ \\
& Stage IIIA & $2.0 \%$ \\
& Stage IIIB & $4.0 \%$ \\
& Stage IIIC & $2.6 \%$ \\
& Stage IV & $0.6 \%$ \\
Ki-67 (proliferation index & Not available & 219 cases \\
& High $(\geq 10 \%)$ & 17
\end{tabular}

Weill Cornell cohort $(n=153)$ and University Hospital Zürich cohort $(n=83)$

†Includes 6 cases from recurrence or metastases, as follows: 1 case of chest wall recurrence; 3 cases of ipsilateral lymph node metastases, 1 case of upper arm metastasis, 1 case of femoral metastasis (Weill Cornell cohort)

*Includes two patients who had bilateral tumours (Weill Cornell cohort).

Juan-Miguel Mosquera ${ }^{1,2 *}$, Sonal Varma ${ }^{1}$, Chantal Pauli ${ }^{1,2,3}$, Theresa. Y. MacDonald ${ }^{1}$, Jossie J. Yashinskie ${ }^{1}$, Zsuzsanna Varga ${ }^{3}$, Andrea Sboner ${ }^{1,2,4}$, Holger Moch ${ }^{3}$, Mark. A. Rubin ${ }^{1,2} \S \&$ Sandra. J. Shin ${ }^{1} \S$

${ }^{1}$ Department of Pathology and Laboratory Medicine, Weill Cornell Medical College, New York, New York, 10065 USA.

${ }^{2}$ Institute for Precision Medicine of Weill Cornell Medical College and New York-Presbyterian Hospital, New York, New York 10021, USA.

${ }^{3}$ Institute for Surgical Pathology, University Hospital Zürich 8091, Switzerland.

${ }^{4}$ Institute for Computational Biomedicine, Weill Cornell Medical College, New York, New York 10021, USA.

email: rubinma@med.cornell.edu

*These authors contributed equally to this work.

$\S$ These authors jointly supervised this work.

Received 17 March 2013; accepted 9 January 2015

Competing Financial Interests: Declared none.

$<\mathrm{jrn}>1$. Banerji, S. et al. Sequence analysis of mutations and translocations across breast cancer subtypes. Nature 486, 405-409 (2012). Medline CrossRef $</$ jrn $>$ 
$<$ jrn>2. Bauer, K. R., Brown, M., Cress, R. D., Parise, C. A. \& Caggiano, V. Descriptive analysis of estrogen receptor (ER)-negative, progesterone receptor (PR)-negative, and HER2-negative invasive breast cancer, the so-called triple-negative phenotype: a population-based study from the California cancer Registry. Cancer 109, 1721-1728 (2007). Medline CrossRef $</$ jrn $>$

$<$ jrn>3. Pal, S. K., Childs, B. H. \& Pegram, M. Triple negative breast cancer: unmet medical needs. Breast Cancer Res. Treat. 125, 627-636 (2011). Medline CrossRef $<$ jrn $>$

$<\mathrm{jrn}>4$. Craig, D. W. et al. Genome and transcriptome sequencing in prospective refractory metastatic triple negative breast cancer uncovers therapeutic vulnerabilities. Mol. Cancer Ther. 12, 104-116 (2013). Medline $</ j \mathrm{jn}>$

$<j \mathrm{jn}>5$. Robinson, D. R. et al. Functionally recurrent rearrangements of the MAST kinase and Notch gene families in breast cancer. Nature Med. 17, 1646-1651 (2011). Medline CrossRef $<$ jrn $>$

$<\mathrm{jrn}>6$. Berger, M. F. et al. The genomic complexity of primary human prostate cancer. Nature 470, 214-220 (2011). Medline CrossRef $<$ jrn $>$

$<$ jrn> 7. Perner, S. et al. TMPRSS2:ERG fusion-associated deletions provide insight into the heterogeneity of prostate cancer. Cancer Res. 66, 8337-8341 (2006). Medline CrossRef $<<$ jrn $>$

$<\mathrm{jrn}>8$. Tomlins, S. A. et al. Recurrent fusion of TMPRSS2 and ETS transcription factor genes in prostate cancer. Science 310, 644-648 (2005). Medline $\underline{\text { CrossRef }</ j r n>}$

$<\mathrm{jrn}>9$. Sboner, A. et al. FusionSeq: a modular framework for finding gene fusions by analyzing paired-end RNA-sequencing data. Genome Biol. 11, R104 (2010). Medline $\underline{\text { CrossRef }}</$ jrn $>$

$<\mathrm{jrn}>$ 10. Pflueger, D. et al. Discovery of non-ETS gene fusions in human prostate cancer using next-generation RNA sequencing. Genome Res. 21, 56-67 (2011). Medline $\underline{\text { CrossRef }}<$ jrn $>$

$<\mathrm{jrn}>11$. Mosquera, J. M. et al. Recurrent NCOA2 gene rearrangements in congenital/infantile spindle cell rhabdomyosarcoma. Genes Chromosom. Cancer 52, 538-550 (2013). Medline $\underline{\text { CrossRef }</ j r n>~}$ 
$<$ jrn>12. Mosquera, J. M. et al. Novel miR143-NOTCH fusions in benign and malignant glomus tumors. Genes Chromosom. Cancer 52, 1075-1087 (2013). Medline CrossRef $</$ jrn $>$

$<$ jrn>13. Dobin, A. et al. STAR: ultrafast universal RNA-seq aligner. Bioinformatics 29, 15-21 (2013). Medline CrossRef $</$ jrn $>$

Author Contributions J.M.M. performed experimental work and wrote paper; S.V. performed experimental work and wrote paper; C.P., T.Y.M., J.J.Y., Z.V. and H. M. performed experimental work; A.S. performed data analysis; S.J.S. performed project planning and revised paper; M.A.R. designed research, performed project planning and revised paper.

Competing Financial Interests Declared none. 
